\title{
Tau neutrinos at DUNE: New strategies, new opportunities
}

\author{
Pedro Machado๑, ${ }^{1, *}$ Holger Schulz $\odot,{ }^{2, \dagger}$ and Jessica Turner®${ }^{1, \sharp}$ \\ ${ }^{1}$ Theoretical Physics Department, Fermi National Accelerator Laboratory, \\ P.O. Box 500, Batavia, Illinois 60510, USA \\ ${ }^{2}$ Department of Physics, University of Cincinnati, Cincinnati, Ohio 45219, USA
}

(Received 9 July 2020; accepted 13 August 2020; published 22 September 2020)

\begin{abstract}
We propose a novel analysis strategy, which leverages the unique capabilities of the DUNE experiment, to study tau neutrinos. We integrate collider physics ideas, such as jet clustering algorithms in combination with machine learning techniques, into neutrino measurements. Through the construction of a set of observables and kinematic cuts, we obtain a superior discrimination of the signal $(S)$ over the background $(B)$. In a single year, using the nominal neutrino beam mode, DUNE may achieve $S / \sqrt{B}$ of 3.3 and 2.3 for the hadronic and leptonic decay channels of the tau respectively. Operating in the tau-optimized beam mode would increase $S / \sqrt{B}$ to 8.8 and 11 for each of these channels. We premier the use of the analysis software RIVET, a tool ubiquitously used by the LHC experiments, in neutrino physics. For wider accessibility, we provide our analysis code.
\end{abstract}

DOI: 10.1103/PhysRevD.102.053010

\section{INTRODUCTION}

Arguably, the tau neutrino is the least understood particle of the Standard Model. Thus far a total of 14 tau neutrinos have been positively identified by the DONuT [1] and OPERA [2] experiments. The former detected beam tau neutrinos from the decay of $D_{s}$ mesons. The latter observed, for the first time, $\nu_{\mu} \rightarrow \nu_{\tau}$ oscillations. In both experiments, the identification of $\tau$ leptons produced by $\nu_{\tau}$ charged current (CC) interactions relies upon the reconstruction of characteristic event topologies: the $\tau$ lepton leaves a millimeter-scale track in the detector emulsion followed by a kink from its subsequent decay. In addition to DONuT and OPERA, the presence of tau neutrinos has been statistically inferred from $\nu_{\mu} \rightarrow \nu_{\tau}$ oscillations of multi-GeV atmospheric neutrinos by Super-Kamiokande [3,4] and IceCube [5]. These searches are based on the $\nu_{\tau}$ contribution to the number of hadronic and/or leptonic neutrino events.

Despite the excellent reconstruction capabilities of DONuT and OPERA and the large statistics of SuperKamiokande and IceCube, tau neutrino observables, such as $\nu_{\tau}$-nuclei cross sections and oscillation parameters extracted from $\nu_{\tau}$ measurements, have large statistical

\footnotetext{
pmachado@fnal.gov

schulzhg@ucmail.uc.edu

\#jturner.@fnal.gov
}

Published by the American Physical Society under the terms of the Creative Commons Attribution 4.0 International license. Further distribution of this work must maintain attribution to the author(s) and the published article's title, journal citation, and DOI. Funded by SCOAP. and systematic uncertainties. Indeed, the tau neutrino nucleon interaction cross section has larger uncertainties [1,4-6] than its electronic [7-9] and muonic [10-12] counterparts. Super-Kamiokande provided the most accurate measurement of the $\nu_{\tau} \mathrm{CC}$ cross section using atmospheric neutrinos and this has an uncertainty of $21 \%$ [4]. Moreover, the unoscillated tau neutrino flux itself is a source of systematic uncertainty as it depends on the $D_{s}$ production rate, the accuracy of which is limited by the incomplete understanding of hadronic effects. These two sources of uncertainty can be mitigated via the direct study of tau neutrino production as proposed by the DsTau experiment [13]. This collaboration aims to provide an independent $\nu_{\tau}$ flux prediction for future neutrino beams with an uncertainty below $10 \%$. As such, the systematic error of the $\mathrm{CC} \nu_{\tau}$ cross section prediction can be lowered. This measurement, together with the use of near-to-far detector ratios, will be crucial in limiting systematic uncertainties in future studies of tau neutrinos at longbaseline neutrino experiments.

The observation of tau neutrinos in current neutrino beams relies on tau appearance due to oscillations. The phase of the oscillation is given by terms such as

$$
\sin ^{2}\left(\frac{\Delta m^{2} L}{4 E}\right) \simeq \sin ^{2}\left(1.27 \frac{\Delta m^{2} / \mathrm{eV}^{2} L / \mathrm{km}}{E_{\nu} / \mathrm{GeV}}\right)
$$

where $\Delta m^{2}$ is a mass squared splitting, $E_{\nu}$ is the neutrino energy and $L$ is the experimental baseline. The larger of the two mass squared splittings is $\Delta m_{\mathrm{atm}}^{2} \simeq 2.5 \times 10^{-3} \mathrm{eV}^{2}$ and the neutrino energy threshold to create a tau lepton from CC interactions is $E_{\nu} \gtrsim 3.5 \mathrm{GeV}$. Therefore, the baseline 
necessary to maximize $\nu_{\tau}$ appearance is of the order 2000 $3000 \mathrm{~km}$. At such long distances, accelerator neutrino experiments require extremely powerful neutrino sources to amass sufficient statistics in order to study the $\nu_{\tau}$ sector. A further difficulty associated with $\nu_{\tau}$ detection is that the decay of a $\tau$ lepton always includes a $\nu_{\tau}$ in the final state which carries away a fraction of undetectable energy. Therefore, reconstruction of the original beam neutrino energy is a challenging task. Although atmospheric neutrinos have energies well above the tau production threshold and travel the necessary distances to induce large $\nu_{\mu} \rightarrow \nu_{\tau}$ oscillations, reconstructing the energy of these neutrinos is challenging as the direction of the incident particle is not known on an event-by-event basis.

Despite such difficulties, upcoming multipurpose neutrino experiments, such as DUNE [14], are well posed to detect tau neutrinos given their large fiducial volume, powerful neutrino beam and exquisite track reconstruction capability. There have been a number of works which explore the tau neutrino sector including pioneering proposals for observing $\nu_{\tau}$ at beam dump experiments [15] and the subsequent experimental search by NOMAD [16], polarization effects on $\tau$ decay products for atmospheric and beam $\nu_{\tau}$ searches [17-21], optimization of cuts on $\tau$ decay products in the DUNE beam neutrino sample [22], tests of unitarity and patterns of leptonic mixing [23-25] and beyond the Standard Model physics probes with $\nu_{\tau}$ measurements [26-29].

In this paper, we are particularly interested in future liquid argon time projection chamber (LArTPC) experiments such as DUNE which have proven to be endowed with excellent event topology reconstruction capabilities. The future DUNE experiment will combine bubble chamber quality data with calorimetry and large statistics. It will therefore provide an unprecedented opportunity to study the $\nu_{\tau}$ sector. We begin in Sec. II with a general discussion of the DUNE experiment and tau neutrino detection at LArTPCs. We perform a sophisticated simulation of the tau neutrino signal and background processes in DUNE, taking into account tau lepton polarization and nuclear physics effects. This is outlined in Sec. III where we build on and expand the analysis performed in Ref. [22] by using modern techniques, such as jet clustering algorithms and deep neural networks, in order to optimize signal-to-background ratios. Furthermore, we quantify the importance of charge identification of pions and running in the tauoptimized beam configuration for tau neutrino searches. Finally, we summarize and discuss future studies, which will incorporate detector effects, in Sec. IV.

\section{TAU NEUTRINOS AT DUNE}

Before discussing tau neutrino events in detail, we first provide a description of the key aspects of the DUNE experiment. The neutrino beam at DUNE is produced by a $120 \mathrm{GeV}$ proton beam hitting a target. The nominal beam power will be approximately $1.2 \mathrm{MW}$ and is expected to

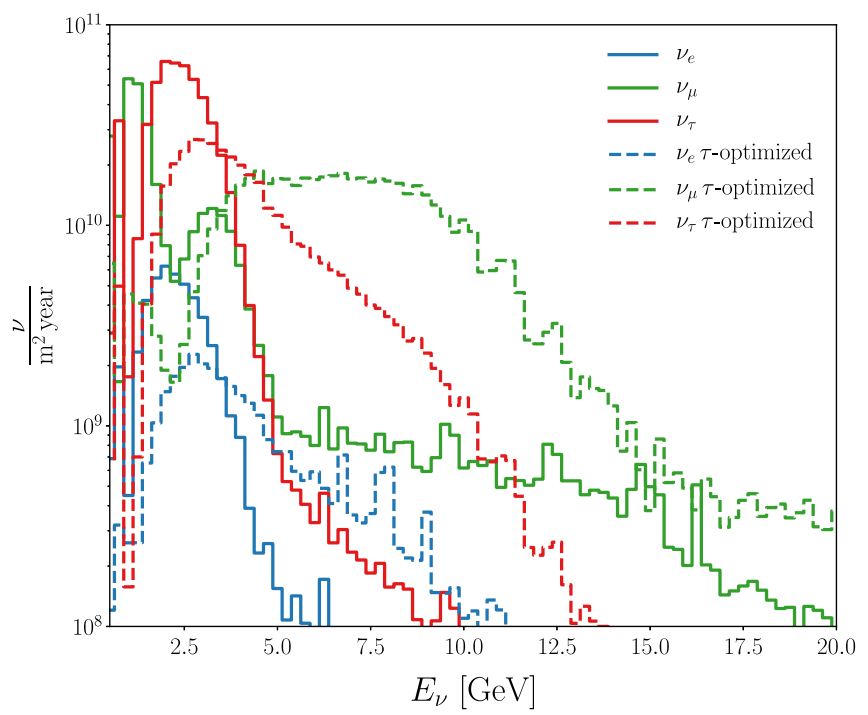

FIG. 1. Nominal (solid) and tau-optimized (dashed) neutrino fluxes at the DUNE far detector neutrino for $\nu_{e}$ (blue), $\nu_{\mu}$ (green) and $\nu_{\tau}$ (red).

deliver $1.1 \times 10^{21}$ protons on target per year. The far detector consists of a 40 kiloton fiducial mass LArTPC with a baseline of $1300 \mathrm{~km}$.

In Fig. 1 we show the oscillated neutrino fluxes at the DUNE far detector for the nominal neutrino mode (solid) and tau-optimized configuration (dashed) for each neutrino flavor [30]. The oscillation parameters chosen as inputs throughout this paper are taken from global fit data [31]:

$$
\begin{aligned}
\Delta m_{21}^{2} & =7.4 \times 10^{-5} \mathrm{eV}^{2}, \quad \Delta m_{31}^{2}=2.5 \times 10^{-3} \mathrm{eV}^{2}, \\
s_{12}^{2} & =0.31, \quad s_{13}^{2}=0.0224, \quad s_{23}^{2}=0.5, \quad \delta_{C P}=1.2 \pi,
\end{aligned}
$$

where $s_{i j} \equiv \sin \theta_{i j}$. In the nominal neutrino mode, the fluxes of all three neutrino flavors peak in the range 1-3 GeV. Therefore many of the tau neutrinos (and antineutrino contaminants) have energies below the tau lepton production threshold. While the integrated flux of the tau-optimized mode is similar to the nominal mode, the peak of the spectra for all three flavors is broader and consequently there are more tau neutrinos with energies above the tau production threshold at the far detector.

In this study, we do not consider $\mu / \pi$ misidentification or energy and angular resolutions. Furthermore, we assume that particles above a certain energy threshold will be identified and reconstructed with 100\% efficiency in the detector [32] as detailed in Sec. III. We acknowledge our approach is optimistic as we do not apply detector effects. Moreover, application of such effects will inevitably deteriorate the signal-to-background ratio; however, the purpose of this paper is to establish a new tool chain and apply novel techniques which will be of use in a more detailed future study.

Conservatively, we assume neutrons are completely invisible at LArTPCs. However, the techniques implemented in 
TABLE I. Dominant decay modes of $\tau^{-}$. All decays involving kaons, as well as other subdominant decays, are in the "Other" category.

\begin{tabular}{lc}
\hline \hline Decay mode & Branching ratio \\
\hline Leptonic & $35.2 \%$ \\
$e^{-} \bar{\nu}_{e} \nu_{\tau}$ & $17.8 \%$ \\
$\mu^{-} \bar{\nu}_{\mu} \nu_{\tau}$ & $17.4 \%$ \\
Hadronic & $64.8 \%$ \\
$\pi^{-} \pi^{0} \nu_{\tau}$ & $25.5 \%$ \\
$\pi^{-} \nu_{\tau}$ & $10.8 \%$ \\
$\pi^{-} \pi^{0} \pi^{0} \nu_{\tau}$ & $9.3 \%$ \\
$\pi^{-} \pi^{-} \pi^{+} \nu_{\tau}$ & $9.0 \%$ \\
$\pi^{-} \pi^{-} \pi^{+} \pi^{0} \nu_{\tau}$ & $4.5 \%$ \\
Other & $5.7 \%$ \\
\hline \hline
\end{tabular}

this work could benefit from information on the energy deposited by secondary hard neutron-proton scattering or multiple neutron scatterings. Such processes can constitute a considerable fraction of the total hadronic energy of an event [33]. Exploring this possibility would require a fully fledged detector simulation which is beyond the scope of this paper.

Finally, we exploit DUNE's capability to identify the charge of pions via the topology of the pion tracks (see e.g., Refs. $[32,34,35])$. Charged pions can undergo many different processes as they travel through a dense medium. These processes include two-body pion absorption $\left(\pi^{+} n p \rightarrow p p, \pi^{-} n p \rightarrow n n\right)$, elastic scattering, single charge exchange $\left(\pi^{+} n \rightarrow \pi^{0} p, \pi^{-} p \rightarrow \pi^{0} n\right)$ and inelastic scattering. Most importantly, stopped $\pi^{-}$are typically captured by the positively charged argon nucleus as opposed to stopped $\pi^{+}$which simply decay to $\mu^{+}$followed by a Michel $e^{+}$. Due to these distinctively different topologies, charge identification of pions is possible on an event-by-event basis. It should be noted there have also been discussions on exploiting topological information [36,37] to statistically distinguish the charge of muons, see e.g., Sec. 5.5.2.1 of Ref. [32]. In principle, similar techniques could be used to infer charge identification of pions statistically [38]. We study the impact of perfect pion charge identification on signal and background yields and contrast this with the case $\pi^{+}$ cannot be distinguished from $\pi^{-}$.

Now that we have discussed the details of the DUNE experiment and the assumptions underlying our analysis, we proceed onto the physics of taus and tau neutrinos. As the DUNE beam is predominantly comprised of muon neutrinos, the main sample of tau neutrinos at the far detector are due to $\nu_{\mu} \rightarrow \nu_{\tau}$ oscillations. At the oscillation minimum of $E_{\nu} \sim 3 \mathrm{GeV}$, the majority of muon neutrinos are expected to have oscillated to tau neutrinos.

A key element in the study of tau neutrino physics is the decay modes of the tau lepton. The most relevant tau branching ratios are given in Table I. In the following, we
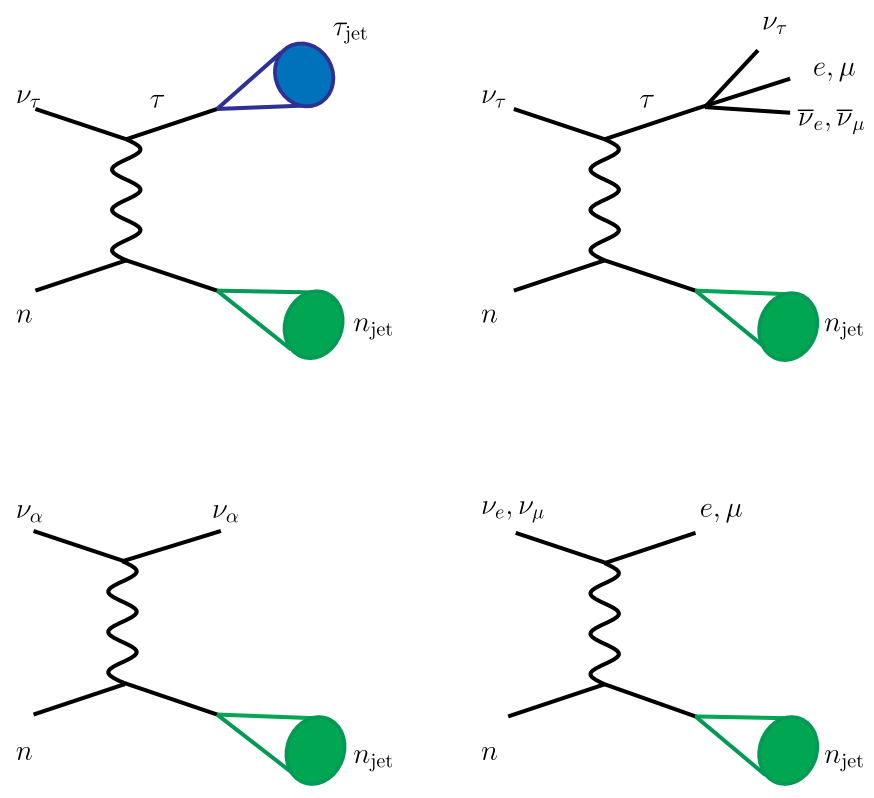

FIG. 2. Pictorial representation of hadronic tau (upper left) and leptonic tau (upper right) signals, and their corresponding backgrounds (lower).

denote the sample of taus that decay to electrons and muons as $\tau_{e}$ and $\tau_{\mu}$ respectively while hadronically decaying taus will be denoted as $\tau_{\text {had }}$. The tau decay length has a value of $c \tau \approx 87 \mu \mathrm{m}$, which is much larger than the argon nuclear radius (of about $3.4 \mathrm{fm}$ ), and thus the tau decays far outside the nucleus. Subsequently, its decay products are not subject to the argon's nuclear potential. However, the tau lifetime is too short to lead to observable displaced vertices in DUNE where the granularity is limited by the typical wire spacing of a few millimeters. It is thus unlikely that tau tracks can be observed at DUNE. ${ }^{1}$ This, together with the severe background, makes $\nu_{\tau}$ detection particularly challenging.

The background of the $\tau_{\mu}$ signal stems mainly from $\nu_{\mu} \mathrm{CC}$ events. ${ }^{2}$ This channel is widely considered to be experimentally intractable as the $\nu_{\mu}$ flux is prohibitively large. Similarly, the dominant background of the $\tau_{e}$ signal are $\nu_{e}$ CC events. As the $\nu_{e}$ flux at DUNE is a small fraction of the total neutrino flux, we study $\nu_{\tau}$ detection in this channel in Sec. III B.

Finally, the dominant background to $\tau_{\text {had }}$ are the neutral current (NC) neutrino scattering events which have contributions from all three neutrino flavors. Despite the fact that all neutrino flavors contribute to $\mathrm{NC}$ events (including tau neutrinos), the NC cross section is smaller than the CC cross section $[39,40]$. Furthermore, the hadronic branching fraction of taus is almost twice as large as the leptonic

\footnotetext{
${ }^{1}$ It is possible a handful of tau tracks could be observed from atmospheric tau neutrinos as they have very high energies.

${ }^{2}$ There is a subdominant contribution from the $\bar{\nu}_{\mu} \mathrm{CC}$ interactions.
} 
branching fraction (see Table I). Consequently, the hadronic decays of the tau have a higher signal-to-background ratio, in the nominal beam mode, than either of the leptonic channels as we outlined in Sec. III A. We note that there is a small contribution to the signal and background from $\bar{\nu}_{\tau}$ $\mathrm{CC}$ and $\bar{\nu}_{e, \mu, \tau} \mathrm{NC}$ events respectively which we include in our analysis.

A pictorial summary of the dominant tau signals and backgrounds is shown in Fig. 2. In the upper right (left) we show the leptonic (hadronic) decay of the tau and in the lower right (left) its associated dominant background. The target nucleon inside the argon nucleus is denoted as $n$ and the green cones represent the (mostly) hadronic activity that emerges from the argon nucleus after the hadronization and subsequent intranuclear cascade. We denote this collection of particles, emerging from the nucleus, as $n_{\text {jet }}$ because we apply a jet clustering algorithm to the signal and background events. Likewise, in the case of the hadronic decays of the tau we represent the final states as $\tau_{\text {jet }}$.

\section{SIMULATION AND RESULTS}

In order to account for nuclear physics effects, the signal and background neutrino-nucleon interactions are simulated using the GiBUU event generator [41]. As discussed before, the signal process is a $\mathrm{CC}$ interaction of a tau neutrino or antineutrino with the argon nucleus. This interaction produces a tau which then decays far outside the argon nucleus. The Monte-Carlo events output by GiBuU factorize into the stable, polarized tau and other final state particles such as pions, protons and neutrons. The latter are products of the propagation or recoiled/created nucleons throughout the nuclear medium and are thus subject to the nuclear potential, rescattering and absorption processes.

The tau lepton produced by a neutrino $\mathrm{CC}$ interaction will be polarized and the distributions of its decay products will critically depend on its spin polarization. Therefore it is important to consider the spin polarization of taus in addition to their production cross sections. This has been discussed at length for tau neutrinos at OPERA [17] and also atmospheric neutrinos at Super-Kamiokande [20] and DUNE [22]. We use the TAUOLA package [42], which decays the tau according to its branching ratios (see Table I) and accounts for tau polarization effects.

We performed our analysis using the RIVET toolkit [43] which is a widely used analysis code for the LHC and other high energy physics collider experiments. However, we find its utility equally applicable to neutrino experiments and in this work we premier its use at DUNE. Tau neutrino interactions typically lead to a high multiplicity of particles in the final state. This is especially the case for hadronically decaying taus which exhibit significant branching fractions to multiple mesons. In our analysis, we apply a jet clustering algorithm to all visible final state particles. These include protons, charged pions, photons (as they lead to electromagnetic showers) and charged kaons but not neutrons or neutrinos. Jet clustering algorithms are an essential tool for a variety of LHC studies; however, we demonstrate their utility for both the $\tau_{\text {had }}$ and $\tau_{e}$ channels. We undertake this treatment for two reasons: first, the physics of individual meson formation depends on the hadronization process, which is largely incalculable, while jets are objects constructed to capture the underlying hard physics which is much better understood. Second, applying a jet clustering algorithm via FastJet [44] is straightforward in RIVET and we demonstrate this technique to be a useful method of characterizing event topologies at LArTPCs.

We divide our analysis into the hadronic and leptonic channels and present them in Secs. III A and III B respectively. We have used $C P$-optimized fluxes derived for the forward horn current polarity (neutrino mode) unless otherwise specified. We will also present DUNE's sensitivity to tau neutrinos in the tau-optimized beam configuration.

\section{A. Hadronic channel}

As discussed, the dominant background to the hadronically decaying taus consists of NC neutrino-argon interactions which receive contributions from all three neutrino flavors. There are contributions from the NC interactions coming from wrong-sign neutrino contaminants but these are subdominant as $\bar{\nu}_{e}, \bar{\nu}_{\mu}$ and $\bar{\nu}_{\tau}$ comprise $0.5 \%, 3.5 \%$ and $3.3 \%$ of the nominal neutrino beam, respectively, in the far detector. The $\bar{\nu}_{\tau} \mathrm{CC}$ interactions also provide a small contribution to the signal and this is included in the analysis of the hadronic channel.

The first step in constructing the analysis for $\tau_{\text {had }}$ is to veto final state particles below the following minimum energy thresholds [14]:

(i) $\pi^{ \pm}: E>100 \mathrm{MeV}$

(ii) $p: E>50 \mathrm{MeV}$

(iii) $\gamma, e, \mu: E>30 \mathrm{MeV}$.

The Monte-Carlo events contain many neutral pions, which have a decay length $c \tau \approx 46.5 \mu \mathrm{m}$. As the decay length is much larger than typical nuclear radii, GiBUU propagates the neutral pions out of the nucleus and does not decay them. On the other hand, this decay length is too small to be resolved by DUNE and thus the $\pi^{0}$ s decay promptly in the detector. We decay the $\pi^{0} \mathrm{~s}$ by boosting them to their rest frame then decaying them isotropically to two photons and boosting the system back to the lab frame. For the $\tau_{\text {had }}$ channel we have analyzed the Monte-Carlo output (as generated by GiBUU and TAUOLA) in terms of average multiplicity and energy sum as shown in Fig. 3. The left (right) plot shows the average multiplicity (energy sum) of visible final state particles as a function of the true neutrino energy. The events which fill these histograms have had the aforementioned thresholds applied to them. The energy threshold for tau production is evident from the signal (in both beam modes) which activates around $E_{\nu} \sim 3 \mathrm{GeV}$. Moreover the initial multiplicity, at low values of $E_{\nu}$, of the signal is $\sim 4$ which corresponds to a few visible hadrons 

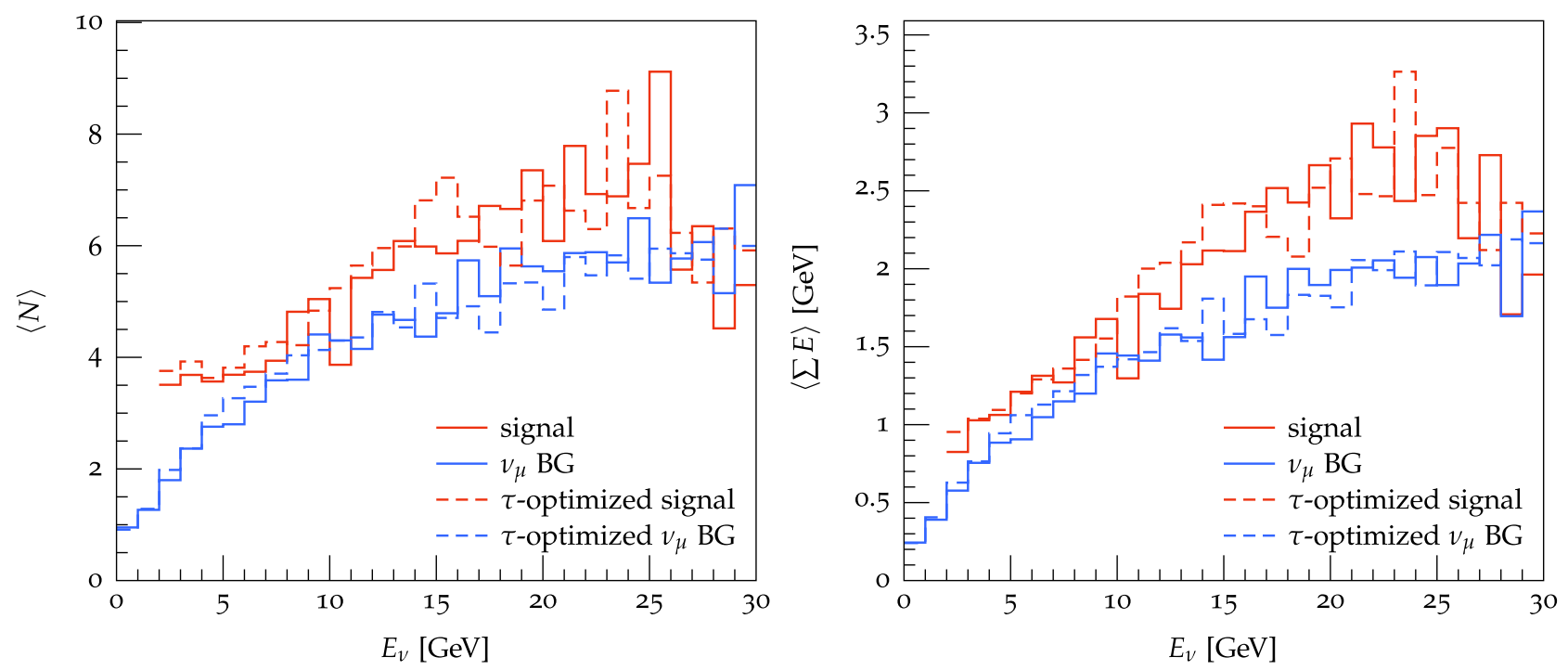

FIG. 3. The left (right) plot shows the mean number (average sum of energies) of visible particles as a function of the true neutrino energy $E_{\nu}$ for the hadronic channel. The signal from the nominal (tau optimized) beam mode is shown in solid (dashed) red and its dominant background in solid (dashed) blue.

emerging from the nuclear cascade combined with the tau dominantly decaying to two visible pions. Unsurprisingly, the multiplicity of both the signal and background grows as a function of the true neutrino energy and is similar at high neutrino energies. From the right plot of Fig. 3 we observe that the average visible energy sum of the signal displays a threshold while the (dominant) background can produce low energy and multiplicity final states. We note that at high values of the true neutrino energy $(12-25 \mathrm{GeV})$ the signal has a larger average visible energy sum than the background. For this regime, in the case of the signal, much of the true neutrino energy will be deposited in the visible final state produced from the tau decays. However, in the case of the background, for the same value of true neutrino energy, all the deposited energy will result in the hadronic shower which can contain invisible neutrons.

In order to optimize the signal-to-background ratio, we study the distributions of several kinematic variables initially assuming charge identification of the pion is possible. Here we provide a list of these variables and the cuts we apply:

(1) $N_{\text {lep }}$ is the number of $e^{ \pm}$and $\mu^{ \pm}$. We veto events containing any such leptons in the final state.

(2) $N_{\pi^{-}}$is the number of $\pi^{-}$'s in the final state. We veto events containing zero $\pi^{-}$.

(3) $\pi_{\text {lead }}^{-}$is the energy of the leading (highest energy) $\pi^{-}$ in each event. We veto events if the leading $\pi^{-}$ has $E<250 \mathrm{MeV}$.

(4) $\sum E_{\text {other }}$ is the total visible energy of the event excluding the leading $\pi^{-}$. We veto events with $\sum E_{\text {other }}<600 \mathrm{MeV}$.

(5) $p_{T}^{\text {miss }}$ is the missing transverse momentum. We veto events with $p_{T}^{\text {miss }}>1 \mathrm{GeV}$.
(6) $N_{\text {jet }}$ is the number of jets in the final state. We veto zero jet events.

We note that the cuts are applied in this order. We also consider the possibility that charge identification is not possible and alter the analysis such that $\pi^{-}$is replaced with $\pi^{ \pm}$.

For the signal, we simulated the tau decaying to all possible final states. Therefore, approximately $35 \%$ of the events contain electrons and muons and the first cut removes this leptonic contribution. To construct the remaining cuts we considered that the dominant tau decays (constituting $\sim 70 \%$ of the hadronic channel) are $\tau^{-} \rightarrow \pi^{-} \nu_{\tau}$, $\tau^{-} \rightarrow \pi^{-} \pi^{0} \nu_{\tau}$ and $\tau^{-} \rightarrow \pi^{-} \pi^{0} \pi^{0} \nu_{\tau}$ as shown in Table I and the signal tends to contain a hard $\pi^{-}$which motivates the second and third cuts. The normalized distributions of the kinematic variables of the signal (red) and electron, muon and tau (blue, green and orange respectively) backgrounds are shown in Fig. 4. We note that cuts have not yet been applied to the events that fill these histograms other than (1) and (2). These distributions show vital shape information that we used to design cuts (3)-(6). The upper left plot shows the normalized distributions of energies of the leading $\pi^{-}$and it is clear that the signal has a larger proportion of high energy $\pi^{-}$while the background has a distinct peak in the low energy bins. For the signal, the hardest $\pi^{-}$originates from the tau decay and it carries $E \gtrsim \mathcal{O}(100) \mathrm{MeV}$ in energy while the background is characterized by many lower energy hadrons resulting from the hadronization process followed by propagation of these hadrons through the nucleus via intranuclear cascade. A crossover between the signal and background distributions occurs around $E_{\pi_{\text {lead }}^{-}} \sim 250 \mathrm{MeV}$ and we place our cut here to enrich the signal and deplete the background. 

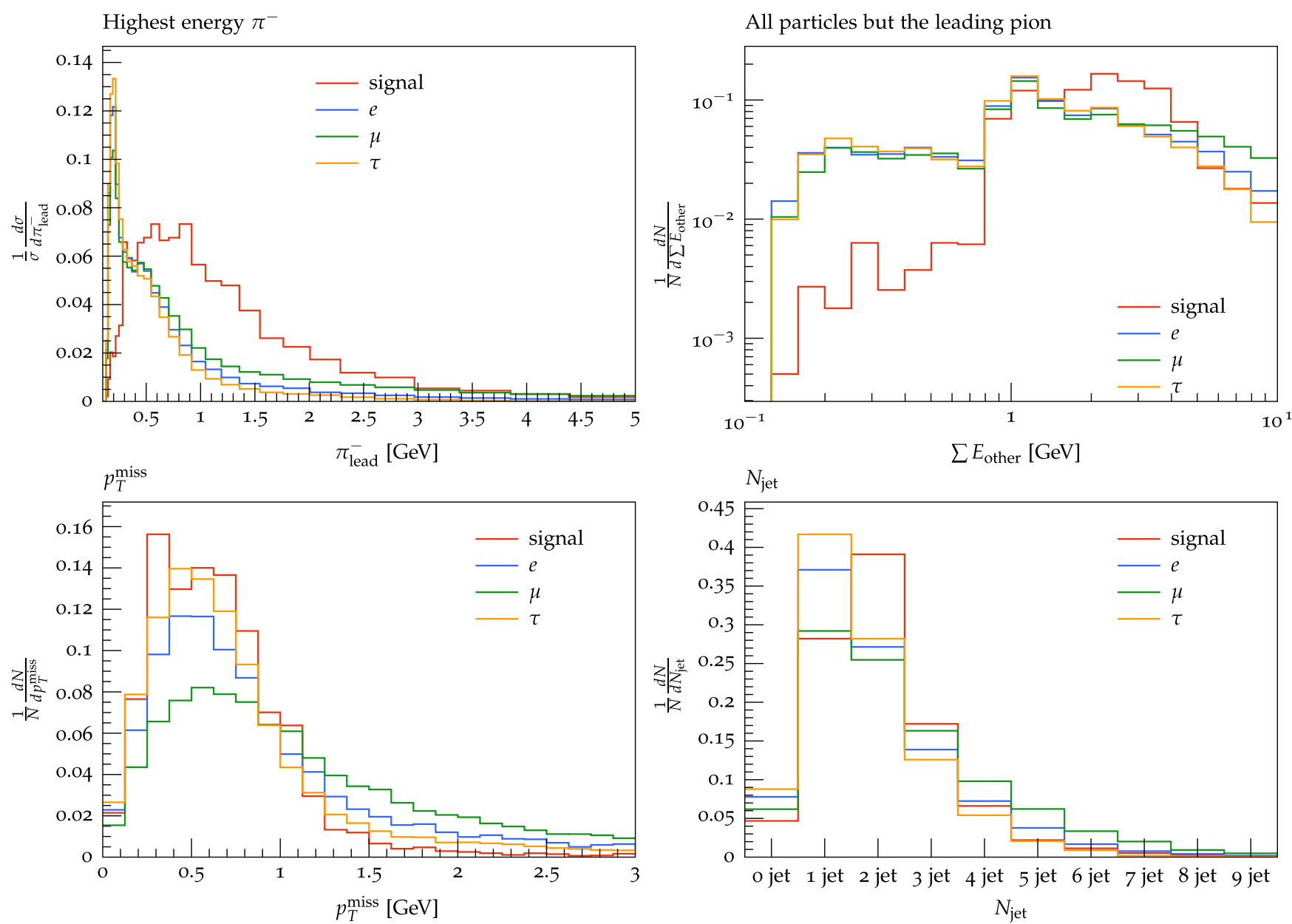

FIG. 4. The normalized distributions of the hadronic signal for the nominal neutrino flux (defined as the CC $\nu_{\tau}$ and $\bar{\nu}_{\tau}$ contribution) and $\nu_{e}, \bar{\nu}_{e}, \nu_{\mu}, \bar{\nu}_{\mu}$ and $\nu_{\tau}, \bar{\nu}_{\tau} \mathrm{NC}$ backgrounds are shown in red, blue, green and orange respectively. In these histograms pion charge identification is assumed.

The second observable we consider is $\sum E_{\text {other }}$ and its corresponding normalized distribution is shown in the upper right plot of Fig. 4. The shape difference between the signal and background is distinct: the background is relatively flat in this observable apart from a slight increase in the distribution around $1 \mathrm{GeV}$. In contrast, the signal distribution has a marked dip below $1 \mathrm{GeV}$. We can observe this dip derives from the tau production threshold as shown in the right plot of Fig. 3 where the average visible energy has an initial value $\sim 1 \mathrm{GeV}$ for true neutrino energies close to the tau production threshold. For $\sum E_{\text {other }}>1 \mathrm{GeV}$ we observe the signal increases and this corresponds to the difference in the signal and background spectra as shown in the right plot of Fig. 3. We note that the reason for this difference is the same in this observable as it was in the aforementioned histogram. We varied the cut in this observable between $200 \leq \sum E_{\text {other }}(\mathrm{MeV}) \leq 800$ and found that a veto on events with $\sum E_{\text {other }}<600 \mathrm{MeV}$ reduces the backgrounds most effectively.
The distribution of the missing transverse momentum is shown in the lower left plot of Fig. 4. The invisible states which contribute to this missing transverse momentum vector are neutrinos and neutrons. ${ }^{3}$ We observe that the signal distribution is more strongly peaked than the background as the former will always have a $\nu_{\tau}$ in the final state. A crossover in the shapes of the signal and background occurs at $p_{T}^{\text {miss }} \sim 900 \mathrm{MeV}$ which motivates cut (5).

The final cut is on the jet multiplicity and we discuss this in more detail due to its nonstandard application in neutrino physics. RIVET uses FastJet to cluster visible final states into jets. We define the jet to have a minimum energy of $1 \mathrm{GeV}$. In particular, we use the Cambridge-Aachen algorithm [45] which falls into the $k_{T}$ class of jet clustering algorithms. In order to be clustered into a single jet, visible particles must be within a radius of $R=0.6$, with $R=\sqrt{\eta^{2}+\phi^{2}}$ where

\footnotetext{
${ }^{3}$ Fermi momentum $\mathcal{O}(200 \mathrm{MeV})$ in the argon nucleus may also contribute to the missing transverse momentum.
} 


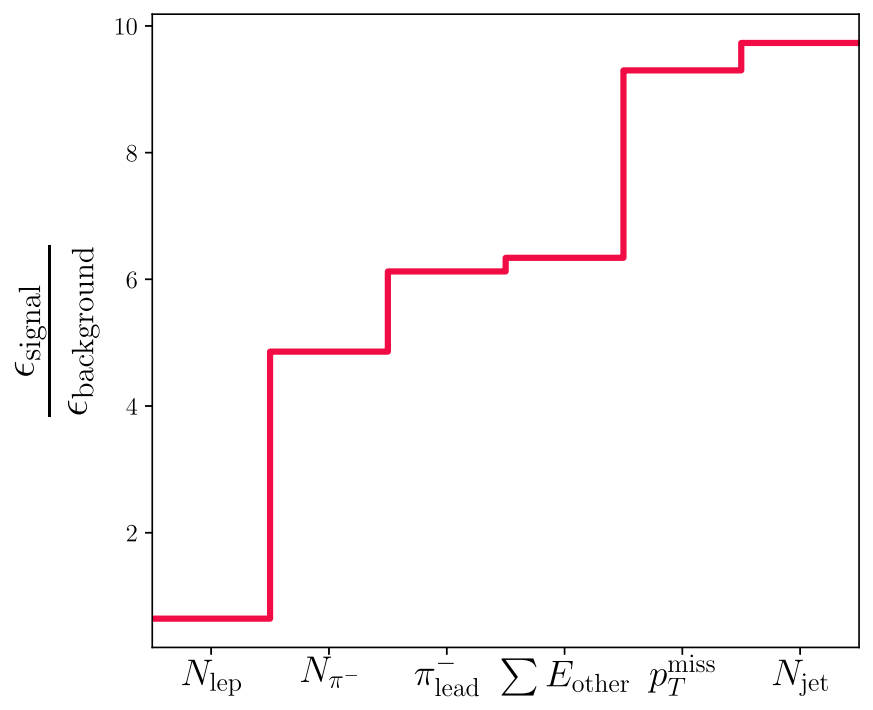

FIG. 5. Cut flow analysis for the hadronic signal and associated NC backgrounds (using the nominal neutrino flux) assuming charge identification of the pion.

$\eta=-\log \tan (\theta / 2)$ is the pseudorapidity ( $\theta$ is the angle between the particle and the jet axis) and $\phi$ is the azimuthal angle with respect to the jet axis. ${ }^{4}$ The lower right plot of Fig. 4 shows the distribution of jet multiplicities. We observe that the signal has a lower zero jet rate than any of the $e, \mu$ or $\tau$ backgrounds. Moreover, the background peaks at one jet while the signal peaks at two jets. We can interpret this in the following way: the background predominantly has a single jet which emerges from the argon nucleus (see the lower left image of Fig. 2 where this jet is denoted as $n_{\text {jet }}$ ). On the other hand, the signal mainly has a two-jet final state where one jet is produced from the tau decay (denoted as $\tau_{\text {jet }}$ in the upper left image of Fig. 2) and another from the intranuclear cascade. The number of jets in the final state can be as high as nine, although these higher jet multiplicities are suppressed. This is due to the broad span of the neutrino beam energy. We found the optimal cut, for this definition of a jet, is to veto zero jet events.

The efficacy of the cuts is summarized in Fig. 5. We show the ratio of efficiencies of the signal over the NC background interactions, as a function of each cut. The application of the cuts to the events should be read from left to right. At this stage, fluxes are not taken into account and this plot simply represents the effectiveness of each cut. The first cut has a value of $\epsilon_{\text {signal }} / \epsilon_{\text {background }} \sim 0.65$ which results from removing the leptons from the signal sample. The second cut, which ensures there is at least one negatively charged pion in the final state, drastically increases the efficiency from $\sim 0.65$ to $\sim 4.8$ and we find

\footnotetext{
${ }^{4}$ We varied the radius in the interval $0.2 \leq R \leq 1.0$ and found the analysis was not particularly sensitive to the jet size in this range.
}

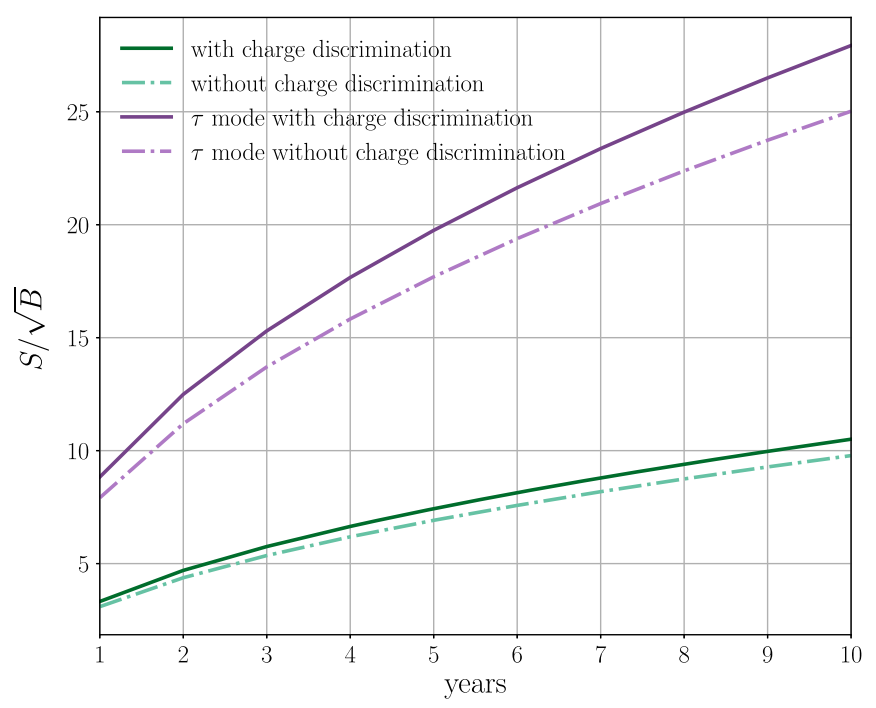

FIG. 6. The solid green (solid purple) shows the signal over square root of background as a function of time for the nominal neutrino (tau optimized) mode assuming pion charge identification. The dot-dashed green (dot-dashed purple) shows the signal over square root of background as a function of time for the nominal neutrino (tau optimized) mode without pion charge identification.

this to be the most aggressive requirement on final states. The cut on the energy of the leading $\pi^{-}$and high values of missing transverse momenta also have a significant effect. Further, we find the requirement of at least a single jet in the final state is more effective than vetoing $\sum E_{\text {other }}<600 \mathrm{MeV}$.

Finally, to demonstrate the impact of these series of cuts on $\nu_{\tau} / \bar{\nu}_{\tau}$ detection at DUNE, we show the significance, defined as $S / \sqrt{B}$ where $S(B)$ is the number of signal (background) events, as a function of run-time in years in Fig. 6. The solid dark green shows the significance for the nominal beam configuration if DUNE has the capability to distinguish $\pi^{-}$from $\pi^{+}$and we find $S / \sqrt{B} \sim 3.3$ within a year of data taking. This corresponds to the detection of 79 and 565 signal and background events respectively. In the scenario that charge identification is not possible, as indicated by dashed light green, the number of signal and background events detected after one year is 83 and 731 respectively. Therefore, the significance decreases to $S / \sqrt{B} \sim 3.1$ and a significance value of 5 requires approximately 2.5 years of data taking. The improvement in the significance with charge identification is mainly due to background reduction, as multi-GeV neutral current interactions will tend to produce comparable amounts of leading $\pi^{+}$and $\pi^{-}$, while the signal is dominated by leading $\pi^{-}$. Thus, pion charge identification can be used to further mitigate backgrounds without reducing the signal. In the optimal scenario, a perfect pion charge discrimination would be equivalent to an increase of about $17 \%$ in exposure in tau neutrino analyses. This result demonstrates 
the nontrivial leverages that LArTPCs may have when making full use of topological information.

We applied the same analysis cuts to the tau-optimized beam sample and found the significance to be almost 3 times higher compared to the nominal beam. The solid dark purple shows the significance with charge discrimination and after one year of data taking is $\sim 8.8$. This corresponds to 433 and 2411 signal and background events respectively. Unsurprisingly, without the charge discrimination capability the significance is lower with a value of $\sim 7.9$ which corresponds to 439 and 3077 signal and background events respectively after the first year of running in tauoptimized mode.

\section{B. Leptonic channel}

As outlined before, the leptonic decay channels of the tau are more challenging than their hadronic counterparts. First, the background cross section (CC interactions from $\nu_{e}$ and $\nu_{\mu}$ ) is larger than the NC background. Second, the tau decays to charged leptons at approximately half the rate as it does to hadrons. Nonetheless, we pursue the $\tau_{e}$ channel where the dominant background is the $\mathrm{CC}$ interaction of electron neutrinos. We neglect the contribution from the $\bar{\nu}_{e}$ CC events as the $\bar{\nu}_{e}$ composition of the neutrino beam is approximately $0.5 \%$ at the far detector. We attempted to construct a simple cut and count analysis for $\tau_{e}$, in a similar manner to the $\tau_{\text {had }}$ analysis, but we found the significance after one year of data taking was below 1.0. In light of this, a more effective way to discriminate the signal from the background is to use a deep neutral net (DNN). In particular, we utilize KERAS with tensorflow [46]. Our methodology is as follows:

(1) Generate signal and background Monte-Carlo samples.

(2) Use RIVET, with the same minimum energy thresholds as before, to calculate kinematic variables or "features" of background and signal events. The signal is assigned a "classification variable" value of 1 and the background 0 .

(3) Separate datasets into training, validation and test samples.

(4) We train the DNN using the binary cross entropy loss function with the training data. We use the validation dataset to guard against overtraining.

(5) We feed the test data through the trained DNN and for each event it returns a "score" between 0 and 1 . If the event is more background-like its score is closer to 0 and conversely if it is more signal-like its score is closer to 1 .

The score can be thought of as a new observable that allows for discrimination of signal and background events. The kinematic variables or features used to characterize the signal and background are

(i) $d \phi_{\min }$ is the minimum angle between the leading (highest energy) $e^{-}$and any other visible particle.

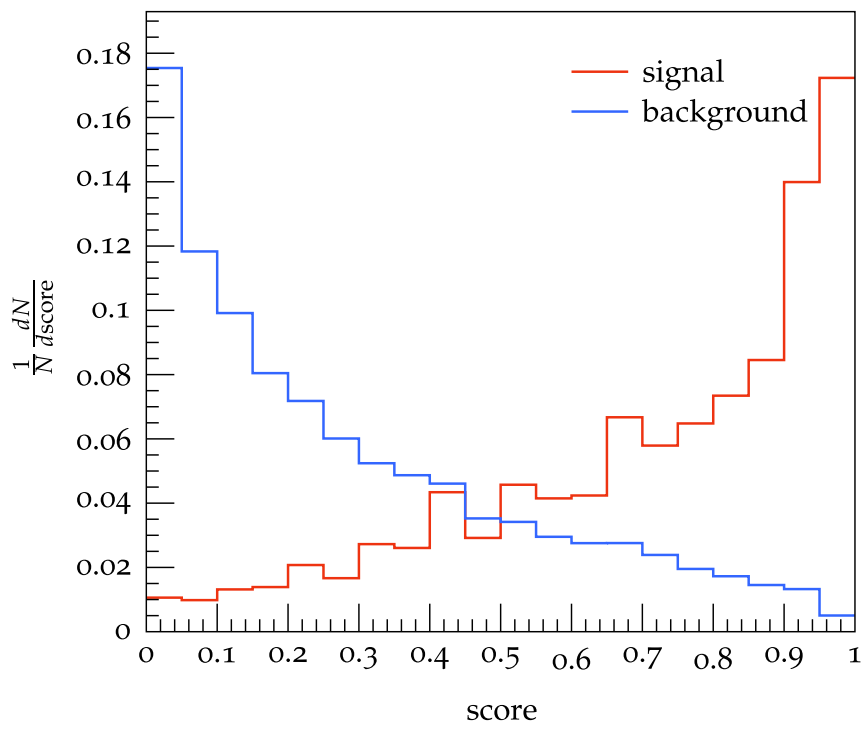

FIG. 7. The normalized distributions of the $\tau_{e}$ signal (red) and associated background (blue) for the nominal neutrino beam.

(ii) $d R_{\min }$ is the $\Delta R$ between leading $e^{-}$and any other visible particle.

(iii) $d \phi_{\text {met }}$ is the $\Delta \phi$ between leading lepton and the missing transverse momentum vector.

(iv) $E_{\text {lead }}^{\ell}$ is the leading lepton energy.

(v) $E_{T}^{\text {miss }}$ is the missing transverse energy.

(vi) $N$ is the number of visible particles other than leading lepton.

(vii) $N_{\pi^{ \pm}}$is the number of $\pi^{ \pm}$'s.

(viii) $\sum E_{\text {other }}$ is the sum of the energies of all visible particles other than leading lepton.

(ix) $\theta_{\ell}$ is the angle of the lepton with respect to the beam axis.

(x) $N_{\text {jet }}$ is the number of jets where jets have the same definition as in the hadronic channel.

(xi) $E_{\text {lead }}^{\text {jet }}$ is the energy of the leading jet.

We note that the lepton is not included in the jet definition and we do not apply charge identification of the pion in this analysis as the number of negatively and positively charged pions should be approximately the same for the $\tau_{e}$ signal and background. Our chosen DNN architecture is a sequential model with a dense input layer followed by two hidden layers of depth 100 sandwiching a dropout layer with a dropout parameter of value 0.2 . The final layer is a sigmoid output layer of depth 1.

The normalized score distributions of signal and background, for nominal neutrino beam mode, are shown in Fig. 7. As expected, the score value of the background peaks at 0 and the signal peaks at 1 . We varied where we placed the cut on the score observable and found vetoing events with a score less than 0.85 yields the highest significance with $S / \sqrt{B}=2.3$ after one year of data taking corresponding to 13 signal events and 32 background events. The significance as a function of time is given 


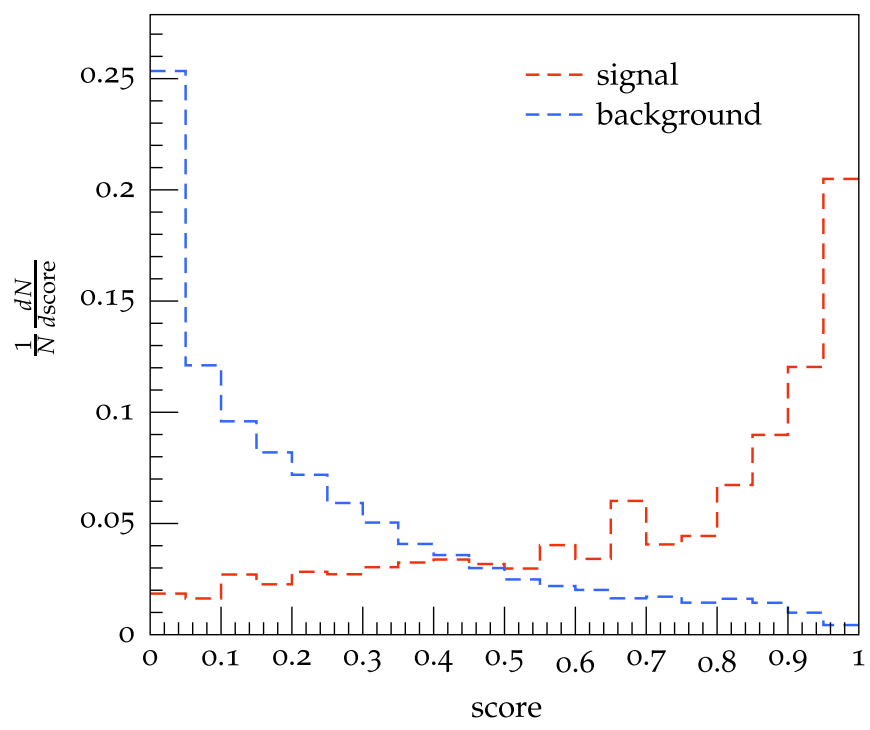

FIG. 8. The normalized distributions of the $\tau_{e}$ signal (red) and associated background (blue) for the tau-optimized neutrino beam.

by the solid dark purple line of Fig. 9 where we observe a $S / \sqrt{B}=5.0$ can be reached after six years of running.

As with the case of the hadronic channel, the detectability of tau neutrinos is vastly improved using the tauoptimized beam. The normalized score distributions of signal and background are shown in Fig. 8. We found that vetoing score values below 0.8 gave us the optimal significance of 11.0 corresponding to 63 signal and 33 background events, respectively, after one year of data taking. The significance is indicated by the dashed pink line of Fig. 9.

Compared to the hadronic tau sample, the $\tau_{e}$ sensitivity using the nominal beam is smaller but may still provide valuable additional information on tau neutrinos. It is remarkable and somewhat surprising that using the tauoptimized beam, the sensitivity of the $\tau_{e}$ sample is comparable to the $\tau_{\text {had }}$ sensitivity (comparing Fig. 6 to Fig. 9). The significant enhancement of $S / \sqrt{B}$ for the $\tau_{e}$ channel in the high energy run can be understood qualitatively by comparing the hadronic and leptonic tau analyses. The backgrounds to $\tau_{\text {had }}$ come from NC events which are flavor blind. Compared to the nominal beam mode, the tau-optimized run presents a higher value of the signal-to-background ratio because there is a larger fraction of the $\nu_{\tau}$ flux above the tau production threshold. This enriches the signal significantly. On the other hand, the $\tau_{e}$ channel receives background contribution mainly from CC $\nu_{e}$ events. This background is strongly affected by oscillations, as the $\nu_{e}$ contamination in the initial neutrino beam is very small. At high energies, $\nu_{\mu} \rightarrow \nu_{e}$ oscillations are suppressed by $1 / E_{\nu}^{2}$, see Eq. (1). Therefore, operating in the tau-optimized mode in the $\tau_{e}$ case not only significantly enriches the signal, but also strongly depletes the

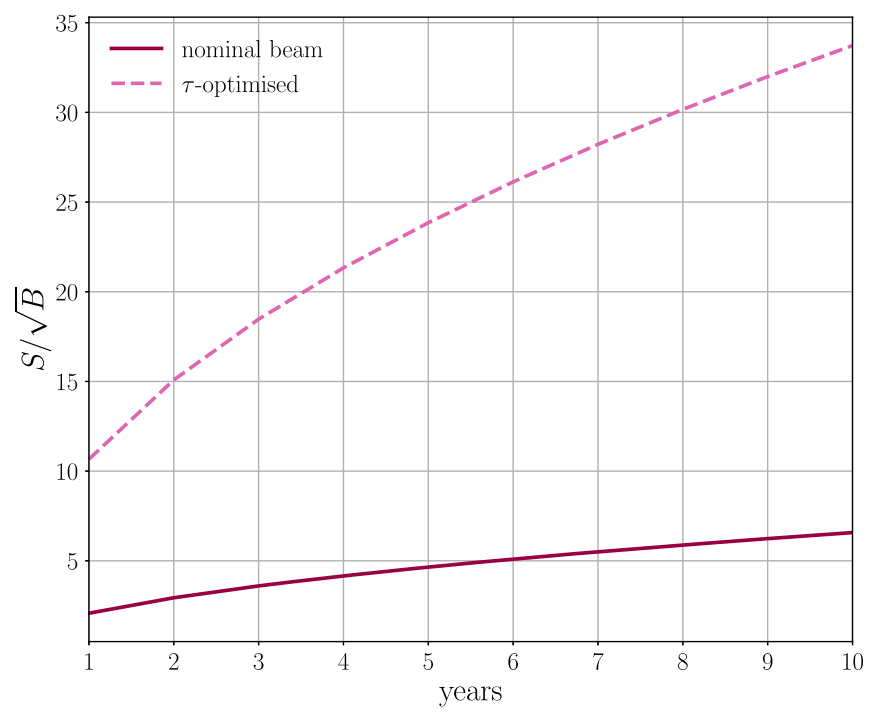

FIG. 9. The solid purple (dashed pink) shows the signal over square root of background as a function of time for the $\tau_{e}$ channel using the nominal (tau-optmized) neutrino flux.

background. This effect can be appreciated in Fig. 1 by comparing the $\nu_{e}$ (blue) and $\nu_{\tau}$ (red) fluxes at the DUNE far detector for the nominal (solid) and tauoptimized (dashed) runs.

\section{DISCUSSION AND FUTURE PROSPECTS}

The analysis strategy proposed in this paper demonstrates the vast potential of LArTPC capabilities. There are two clear avenues for further exploration. First, although we performed an in-depth physics analysis of tau neutrinos at DUNE, a realistic simulation of detector effects is required to completely demarcate DUNE's sensitivity to beam $\nu_{\tau}$ appearance. We intend to explore, in a forthcoming work, the impact of detector effects on the kinematic variables used here and consequently the signalto-background ratio. Second, in order to perform a more robust physics analysis with the tool chain we developed here, a detailed understanding of tau neutrino energy reconstruction is needed in addition to the impact of systematic uncertainties on the inferred $\nu_{\tau}$ spectrum.

Special attention should be given to $\mu^{ \pm} / \pi^{ \pm}$misidentification which can enlarge the $\tau_{\text {had }}$ background. Moreover, $e / \gamma$ separation is needed to reject certain $\mathrm{NC}$ events as a background in the $\tau_{e}$ analysis. This analysis, excluding the missing transverse momentum information, could also be implemented in multi-GeV atmospheric neutrino searches in DUNE and Hyper-Kamiokande [47], with some tuning of the analysis cuts due to much higher incoming neutrino energy.

Ultimately, several physics analyses could benefit from applying our tool chain with realistic detector effects and a treatment of systematic uncertainties. Amongst those are $\nu_{\tau}$-nucleus interaction measurements [6,21]; violations of 
TABLE II. The number of signal and background events after one year of data taking in the hadronic $\left(\tau_{\text {had }}\right)$ and leptonic $\left(\tau_{e}\right)$ channels for nominal and tau-optimized beam modes.

\begin{tabular}{lccccc}
\hline \hline Mode & Beam & Charge id & $N_{\text {sig }}$ & $N_{\text {bg }}$ & $S / \sqrt{B}$ \\
\hline$\tau_{\text {had }}$ & Nominal & $\checkmark$ & 79 & 565 & 3.3 \\
$\tau_{\text {had }}$ & Nominal & $\boldsymbol{X}$ & 83 & 731 & 3.1 \\
$\tau_{\text {had }}$ & tau-optimized & $\checkmark$ & 433 & 2411 & 8.8 \\
$\tau_{\text {had }}$ & tau-optimized & $\boldsymbol{X}$ & 439 & 3077 & 7.9 \\
$\tau_{e}$ & tau-optimized & $\boldsymbol{X}$ & 63 & 33 & 11.0 \\
$\tau_{e}$ & Nominal & $\boldsymbol{X}$ & 13 & 32 & 2.3 \\
\hline \hline
\end{tabular}

unitarity in the leptonic mixing matrix $[23,25,48-50]$; nonstandard neutrinos interactions in neutrino production, detection and propagation [51]; sterile neutrino searches [52-55]; and general consistency tests of the three neutrino oscillation paradigm $[24,28]$. In our work we demonstrate that the tau-optimized beam significantly enhances the prospects of tau neutrino measurements at DUNE. In particular, running in this mode improves the detectability of tau neutrinos in the leptonic channel. A successful case of a high energy neutrino beam run can be found in the MINOS experiment history. For example, the constraints set by MINOS+, the high energy run of MINOS, on eV-scale sterile neutrinos [56] and large extra dimension models [57] remain leading the field even in the presence of newer experiments.

\section{CONCLUSIONS}

We have proposed a novel analysis strategy to study tau neutrinos with the DUNE experiment. The marriage of collider tools and neutrino physics allows us to exploit topological features in neutrino events and thereby significantly extend DUNE's physics reach. The RIVET analysis code used for the hadronic channel is available at [58]. Our results are summarized in Table II and we find that, in the nominal neutrino beam, DUNE could achieve $S / \sqrt{B}=5$ in just over two years of data taking using only hadronically decaying taus or in six years of data taking with leptonically decaying taus. In the tau-optimized beam mode, after a single year of data taking, DUNE could achieve $S / \sqrt{B} \sim 9,11$ for the $\tau_{\text {had }}$ and $\tau_{e}$ channels respectively. Moreover, identifying the pion charge via pion track topology in DUNE would be equivalent to an increase between $17 \%$ and $24 \%$ in exposure.

\section{ACKOWLEDGMENTS}

We thank Kai Gallmeister, Ulrich Mosel and Zbigniew Andrzej Was for their invaluable advice on the usage of GiBUU and TAUOLA. We are grateful to André de Gouvêa for careful reading of the manuscript. We thank Kirsty Duffy, Lukas Heinrich, Joshua Isaacson, Xiangyang Ju, Kevin Kelly, Claudius Krause, Shirley Li, Nhan Tranh, Raquel Castillo Fernandez and Tingjun Yang for helpful discussions on various aspects of this work. This research was supported by the Fermi National Accelerator Laboratory (Fermilab), a U.S. Department of Energy, Office of Science, HEP User Facility. Fermilab is managed by Fermi Research Alliance, LLC (FRA), acting under Contract No. DE-AC0207CH11359. This material is based upon work supported by the U.S. Department of Energy, Office of Science, Office of Advanced Scientific Computing Research, Scientific Discovery through Advanced Computing (SciDAC) program, grant HEP Data Analytics on HPC, No. 1013935. It was supported by the U.S. Department of Energy under Contract No. DE-AC02-76SF00515.
[1] K. Kodama et al. (DONuT Collaboration), Phys. Rev. D 78, 052002 (2008).

[2] N. Agafonova et al. (OPERA Collaboration), Phys. Rev. Lett. 120, 211801 (2018); 121, 139901(E) (2018).

[3] K. Abe et al. (Super-Kamiokande Collaboration), Phys. Rev. Lett. 110, 181802 (2013).

[4] Z. Li et al. (Super-Kamiokande Collaboration), Phys. Rev. D 98, 052006 (2018).

[5] M. G. Aartsen et al. (IceCube Collaboration), Phys. Rev. D 99, 032007 (2019).

[6] Y. S. Jeong and M. H. Reno, Phys. Rev. D 82, 033010 (2010).

[7] J. Blietschau et al. (Gargamelle Collaboration), Nucl. Phys. B133, 205 (1978).

[8] J. Wolcott et al. (MINERvA Collaboration), Phys. Rev. Lett. 116, 081802 (2016).
[9] R. Acciarri et al. (ArgoNeuT Collaboration), Phys. Rev. D 102, 011101 (2020).

[10] K. A. Olive et al. (Particle Data Group), Chin. Phys. C 38, 090001 (2014).

[11] P. Abratenko et al. (MicroBooNE Collaboration), arXiv:2006 .00108.

[12] A. Filkins et al. (MINERvA Collaboration), Phys. Rev. D 101, 112007 (2020).

[13] S. Aoki et al. (DsTau Collaboration), J. High Energy Phys. 01 (2020) 033.

[14] B. Abi et al. (DUNE Collaboration), arXiv:2002.02967.

[15] C. H. Albright and R. E. Shrock, Phys. Lett. 84B, 123 (1979).

[16] P. Astier et al. (NOMAD Collaboration), Nucl. Phys. B611, 3 (2001). 
[17] K. Hagiwara, K. Mawatari, and H. Yokoya, Nucl. Phys. B668, 364 (2003); B701, 405(E) (2004).

[18] J.-M. Levy, J. Phys. G 36, 055002 (2009).

[19] K. Hagiwara, K. Mawatari, and H. Yokoya, Nucl. Phys. B, Proc. Suppl. 139, 140 (2005).

[20] M. Aoki, K. Hagiwara, K. Mawatari, and H. Yokoya, Nucl. Phys. B727, 163 (2005).

[21] J. E. Sobczyk, N. Rocco, and J. Nieves, Phys. Rev. C 100, 035501 (2019).

[22] J. Conrad, A. de Gouvêa, S. Shalgar, and J. Spitz, Phys. Rev. D 82, 093012 (2010).

[23] S. Parke and M. Ross-Lonergan, Phys. Rev. D 93, 113009 (2016).

[24] P. B. Denton, arXiv:2003.04319.

[25] S. A. R. Ellis, K. J. Kelly, and S. W. Li, arXiv:2004.13719.

[26] A. Rashed and A. Datta, Int. J. Mod. Phys. A 32, 1750060 (2017).

[27] D. Meloni, Phys. Lett. B 792, 199 (2019).

[28] A. de Gouvêa, K. J. Kelly, G. V. Stenico, and P. Pasquini, Phys. Rev. D 100, 016004 (2019).

[29] F. Kling, Phys. Rev. D 102, 015007 (2020).

[30] Dune fluxes, https://home.fnal.gov/ ljf26/DUNEFluxes/.

[31] I. Esteban, M. C. Gonzalez-Garcia, A. Hernandez-Cabezudo, M. Maltoni, and T. Schwetz, J. High Energy Phys. 01 (2019) 106.

[32] B. Abi et al. (DUNE Collaboration), arXiv:2002.03005.

[33] A. Friedland and S. W. Li, Phys. Rev. D 99, 036009 (2019).

[34] I. Nutini, Study of charged particles interaction processes on Ar in the $0.2-2.0 \mathrm{GeV}$ energy range through combined information from ionization free charge and scintillation light, Master's Thesis, Florence University, 2015.

[35] E. Gramellini, Measurement of the negative pion and positive kaon total hadronic cross sections on argon at the LArIAT experiment, Ph.D. Thesis, Yale University, 2018.

[36] T. Suzuki, D. F. Measday, and J. Roalsvig, Phys. Rev. C 35, 2212 (1987).

[37] R. Acciarri et al. (MicroBooNE Collaboration), J. Instrum. 12, P09014 (2017).

[38] K. Duffy, Microboone capabilities, measurements and plans, in nuSTEC Workshop on Neutrino-Nucleus Pion Production in the Resonance Region (2019).
[39] G. P. Zeller et al. (NuTeV Collaboration), Phys. Rev. Lett. 88, 091802 (2002); 90, 239902(E) (2003).

[40] J. A. Formaggio and G. P. Zeller, Rev. Mod. Phys. 84, 1307 (2012).

[41] O. Buss, T. Gaitanos, K. Gallmeister, H. van Hees, M. Kaskulov, O. Lalakulich, A. B. Larionov, T. Leitner, J. Weil, and U. Mosel, Phys. Rep. 512, 1 (2012).

[42] S. Jadach, Z. Was, R. Decker, and J. H. Kuhn, Comput. Phys. Commun. 76, 361 (1993).

[43] C. Bierlich et al., SciPost Phys. 8, 026 (2020).

[44] M. Cacciari, G. P. Salam, and G. Soyez, Eur. Phys. J. C 72, 1896 (2012).

[45] Y. L. Dokshitzer, G. D. Leder, S. Moretti, and B. R. Webber, J. High Energy Phys. 08 (1997) 001.

[46] F. Chollet et al., KERAS, https://github.com/fchollet/keras.

[47] K. Abe et al. (Hyper-Kamiokande Collaboration), arXiv: 1805.04163.

[48] Y. Farzan and A. Yu. Smirnov, Phys. Rev. D 65, 113001 (2002).

[49] H.-J. He and X.-J. Xu, Phys. Rev. D 89, 073002 (2014).

[50] X. Qian, C. Zhang, M. Diwan, and P. Vogel, arXiv:1308 .5700 .

[51] P. S. B. Dev et al., SciPost Phys. Proc. 2, 001 (2019).

[52] G. H. Collin, C. A. Argelles, J. M. Conrad, and M. H. Shaevitz, Nucl. Phys. B908, 354 (2016).

[53] S. Gariazzo, C. Giunti, M. Laveder, and Y. F. Li, J. High Energy Phys. 06 (2017) 135.

[54] M. Dentler, Á. Hernndez-Cabezudo, J. Kopp, P. A. N. Machado, M. Maltoni, I. Martinez-Soler, and T. Schwetz, J. High Energy Phys. 08 (2018) 010.

[55] A. Ghoshal, A. Giarnetti, and D. Meloni, J. High Energy Phys. 12 (2019) 126.

[56] P. Adamson et al. (MINOS+Collaboration), Phys. Rev. Lett. 122, 091803 (2019).

[57] P. Adamson et al. (MINOS Collaboration), Phys. Rev. D 94, 111101 (2016).

[58] P. Machado, H. Schulz, and J. Turner, Analysis code supplement to "Tau neutrinos at DUNE: New strategies, new opportunities," https://doi.org/10.5281/zenodo.3924078, version 1.0. 